\title{
ASSOCIAÇÃO DE ESTRESSE OCUPACIONAL E USO DE PSICOTRÓPICOS POR DOCENTES DA ÁREA DA SAÚDE
}

\author{
Association between occupational stress and use of psychotropic drugs by health \\ faculty
}

\section{Asociación entre el estrés ocupacional y el uso de psicotrópicos por docentes del área de la salud}

\author{
lel Marciano de Moraes Filho iD \\ Universidade Paulista - UNIP - Brasília - (DF) - Brasil
}

Caroline Carla de Sousa Dias iD

Faculdade de Ciências e Educação Sena Aires - FACESA - Valparaiso de Goiás (GO) - Brasil

Leonardo Luiz Pinto iD

Faculdade de Ciências e Educação Sena Aires - FACESA - Valparaiso de Goiás (GO) - Brasil

Osmar Pereira dos Santos (iD

Faculdade União de Goyazes - FUG - Trindade (GO) - Brasil

\section{Keila Cristina Félis (iD}

Centro Universitário de Goiatuba - UNICERRADO - Goiatuba (GO) - Brasil

Maria Fernanda Rocha Proença

Faculdade de Ciências e Educação Sena Aires - FACESA - Valparaiso de Goiás (GO) - Brasil

Débora Dadiani Dantas Cangussu iD

Centro Universitário Estácio - Estácio - Taguatinga (DF) - Brasil

Rodrigo Marques da Silva (iD

Faculdade de Ciências e Educação Sena Aires - FACESA - Valparaiso de Goiás (GO) - Brasil

\section{RESUMO}

Objetivo: Analisar a associação entre o nível de estresse ocupacional e uso de psicotrópicos por docentes da área de saúde. Métodos: Estudo transversal, analítico e quantitativo, realizado entre novembro e dezembro de 2018, em uma instituição privada do estado de Goiás, onde 48 docentes universitários da área de saúde responderam a questionários sociodemográfico e profissional, Escala de Estresse no Trabalho e questionário sobre o uso de psicotrópicos. Analisaram-se os dados por meio de medidas descritivas e teste de qui-quadrado. Resultados: Foi detectado alto nível de estresse ocupacional em 39,6\% da amostra. Houve associação significativa entre estresse ocupacional e as seguintes variáveis: uso atual ou prévio de medicação psicotrópica $(0,037)$, percepção de melhora da qualidade de vida após terapia medicamentosa $(p=0,041)$ e realização de atividades de risco no ambiente de trabalho $(p=0,036)$. Conclusão: $O$ uso de psicotrópicos pelos docentes universitários está significativamente associado ao estresse ocupacional na amostra em questão.

Descritores: Psicotrópicos; Docentes; Estresse Ocupacional; Educação Superior; Programa de Saúde Ocupacional.

\section{ABSTRACT}

Objective: To analyze the association between occupational stress levels and use of psychotropic drugs by health faculty. Methods: This quantitative analytical cross-sectional study was conducted from November to December 2018 at a private institution in the state of Goiás where 48 health professors answered a sociodemographic and occupational questionnaire, the workplace stress scale and a questionnaire on the use of psychotropic drugs. Data were analyzed using descriptive measures and the Chi-squared test. Results: High levels of occupational stress were found in $39.6 \%$ of the sample. Occupational stress was significantly associated with the following variables: current or previous use of psychotropic drugs (0.037), perception of quality of

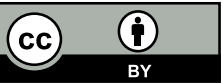


life improvement after drug therapy $(p=0.041)$ and risky activities at work $(p=0.036)$. Conclusion: The use of psychotropic drugs by university professors was significantly associated with occupational stress in the sample analyzed.

Descriptors: Psychotropic Drugs; Faculty; Occupational Stress; Education, Higher; Occupational Health Program.

\section{RESUMEN}

Objetivo: Analizar la asociación entre el nivel de estrés ocupacional y el uso de psicotrópicos por docentes del área de la salud. Métodos: Estudio transversal, analítico y cuantitativo realizado entre noviembre y diciembre de 2018 en una institución privada del estado de Goiás de la cual 48 docentes universitarios del área de la salud han contestado a cuestionarios sociodemográfico y profesional, Escala de Estrés Laboral y cuestionario sobre el uso de psicotrópicos. Se analizaron los datos a través de las medidas descriptivas y de la prueba chi-cuadrado. Resultados: Se ha detectado alto nivel de estrés laboral en el 39,6\% de la muestra. Hubo asociación significante entre el estrés laboral y las variables: uso actual o previo de medicación psicotrópica $(0,037)$, percepción de la mejora de la calidad de vida después de la terapia medicamentosa $(p=0,041)$ y la realización de actividades de riesgo en el ambiente laboral $(p=0,036)$. Conclusión: El uso de psicotrópicos por los docentes universitarios asociase de manera significante al estrés laboral en la muestra investigada.

Descriptores: Psicotrópicos; Docentes; Estrés Laboral; Educación Superior; Programa de Salud Laboral.

\section{INTRODUÇÃO}

Em 2004, no Brasil, fora criado o Sistema Nacional de Avaliação do Ensino Superior, para avaliar as Instituições de Ensino Superior em três dimensões: organização didática-pedagógica; infraestrutura; corpo docente e tutorial ${ }^{(1,2)}$.

No que tange ao corpo docente, as instituições de ensino superior também são avaliadas a partir da titulação e formação dos profissionais e, ainda, das suas ações, baseadas na tríade ensino, pesquisa e extensão, adjunto a produção científica acadêmica, que está diretamente interligada ao trabalho educacional desenvolvido pelos professores, e que, conexos aos outros indicadores, comporão a nota conceitual do curso superior ${ }^{(2,3)}$.

A pressão para comprovação de eficiência do profissional para perfazer os métodos de avaliações institucionais ocasiona estresse no docente. Dessa forma, as primeiras definições de estresse datam de meados dos anos cinquenta, e classificaram o estresse, inicialmente, como uma resposta neuroendócrina não específica do organismo. Mais tarde, porém, fora considerado uma resposta que envolvia outros sistemas do corpo humano, principalmente cardiovascular, pulmonar e renal. Tais sistemas, quando afetados, sugerem um grande risco para o desenvolvimento de doenças que impedem ou limitam a permanência do trabalhador no ambiente de trabalho. Com isso, o estresse se torna um problema de saúde pública ${ }^{(1,4)}$.

Essa condição de pressão pode levar a exaustão física e emocional, que se retrata, principalmente, na saúde mental do profissional docente que apresenta índices elevados de estresse, instalando um ciclo em que atividades docentes associadas aos fatores estressores sociais cotidianos os levam a apatia, conformação e acomodação, que resulta em insatisfação e frustração ${ }^{(5,6)}$.

Quando o docente do ensino superior é da área da saúde, o problema é mais denso, pois o profissional não possui, em sua formação de base, matérias que são voltadas para a docência, além de muitos virem de uma atividade assistencial, ou seja, prática clínica baseada no empirismo, gerando, muitas vezes, estresse na transição nas práxis da difusão educacional( ${ }^{(7)}$.

A busca por soluções imediatas de problemas que afetam o cotidiano das pessoas é um fator que faz o tratamento medicamentoso aparentemente uma alternativa rápida, eficaz e resolutiva na solução de conflitos cotidianos, em oposição aos desafios de um tratamento psicológico, prolongado e "doloroso", pois colocará o indivíduo como protagonista de sua melhora global através do enfrentamento das premissas cotidianas e dos fatores estressores. Dessa forma, a utilização de medicamentos psicotrópicos tem aumentado mundialmente nas últimas décadas ${ }^{(7)}$.

As substâncias psicotrópicas agem nas funções do sistema nervoso central (SNC). A partir de estímulos, através dos seus órgãos do sentido, a informação chega ao SNC, sendo processada, interpretada, elaborada, memorizada, e realizando associações, entre outros. Todos esses processamentos ocorrem em questão de milésimos de segundo e são repetidos milhares de vezes ${ }^{(7,8)}$. Desse mesmo modo, de acordo com o tipo de ação, as drogas psicotrópicas podem provocar diversos tipos de efeitos, como ansiedade, sonolência, excitação e convulsão, que vão de oposição ao bem-estar momentâneo que podem ocasionar ${ }^{(8)}$.

O docente enfrenta notável carga laboral, acúmulo de vínculos de trabalho, desvalorização da profissão e cobranças excessivas - fortalecidas pelos métodos de avaliação curriculares brasileiros ${ }^{(7,8)}$. Frente à possibilidade 
de adoecimento físico e mental do docente, espera-se impacto negativo na sua produtividade no trabalho e no aprendizado dos discentes, o que pode levar ao absenteísmo, presenteísmo e sobrecarregar a equipe de professores que permanecem no local do trabalho. Assim, entendendo a promoção de saúde como o conjunto de políticas, planos e programas de saúde pública cujas ações buscam evitar que as pessoas se exponham a fatores condicionantes e determinantes de doenças, é imprescindível que as condições que levam ao adoecimento do docente e suas consequências sejam analisadas para posterior desenvolvimento de ações preventivas no campo da educação superior ${ }^{(7,8)}$.

Além disso, se faz necessária a implementação de ações preventivas e de promoção da saúde no ambiente de trabalho no que tange ao estresse ocupacional e seus efeitos na vida dos docentes. Dessa forma, o estudo se justifica devido à alta demanda de estresse instalada nos docentes, que leva ao adoecimento ocupacional e, logo, ao sofrimento moral, uma vez que são os multiplicadores de conhecimento e são de fundamental importância para a formação de novos profissionais que atuarão na área da saúde. Se estiveram adoecidos, como proporcionarão uma concepção profissional aos discentes que estão embarcando na carreira profissional e, em breve, no mercado de trabalho?

Nesse sentido, o objetivo deste estudo foi analisar a associação entre o nível de estresse ocupacional e o uso de psicotrópicos por docentes da área de saúde.

\section{MÉTODOS}

Trata-se de um estudo transversal, analítico e quantitativo realizado em uma universidade privada de ensino do estado de Goiás entre novembro e dezembro de 2018.

Foram incluídos docentes vinculados à instituição no período de coleta de dados, atuantes nos cursos da área de saúde (Enfermagem, Farmácia ou Fisioterapia). Sendo excluídos aqueles que atuam somente em estágios (sem inserção no ensino teórico), que faziam uso prévio de psicotrópicos e em férias ou em licença de qualquer natureza.

Os dados foram coletados por meio dos seguintes instrumentos autoaplicáveis: questionário para descrever o perfil sociodemográfico e profissional dos docentes, escala de estresse no trabalho $(E E T)^{(9)}$ e questionário sobre o uso de psicotrópicos. Distribuíram-se esses instrumentos no ambiente de trabalho, mas para serem respondidos no domicílio, após a exposição dos objetivos da pesquisa e a assinatura do Termo de Consentimento Livre e Esclarecido (TCLE). A data de devolução foi agendada com cada docente, segundo sua disponibilidade, pelos pesquisadores previamente treinados para a coleta.

Os questionários sociodemográfico e profissional, construídos pelos autores, envolveram as seguintes variáveis: data de nascimento; sexo; estado civil; presença de filhos; formação acadêmica; curso principal em que atua; categoria profissional exercida na área da saúde; tempo de atuação, de serviço em docência e na instituição de ensino superior; grau de escolaridade; carga horária semanal; regime de trabalho (estatuário, temporário, celetista e voluntário); número de vínculos empregatícios; jornada de trabalho diária; renda mensal total recebida em salários mínimos; satisfação profissional; e percepção de risco quanto à prática laboral.

A EET, construída e validada em $2004^{(9)} \mathrm{com} 437$ trabalhadores de diferentes organizações, públicas e privadas, para mensurar o nível de estresse ocupacional geral, é composta por 23 itens dispostos em escala tipo Likert de cinco pontos, em que: 1 - discordo totalmente, 2 - discordo, 3 - concordo em parte, 4 - concordo e 5 - concordo totalmente. A partir da soma das pontuações assinaladas em cada item, obtêm-se os escores de estresse ocupacional, sendo que, quanto maior a pontuação, maior o estresse apresentado pelo indivíduo no âmbito do trabalho. A partir da média geral para a população pesquisada, o estresse é dicotomizado em baixo (quando o indivíduo apresenta escore de estresse inferior à média da população) e alto estresse (quando o indivíduo apresenta escore de estresse superior à média da população). Os itens de maior média representam as situações que representam maior estresse aos docentes. No processo de validação, foram observados alfa de Cronbach de 0,91 para o grupo de 23 itens do instrumento ${ }^{(9)}$.

O questionário para avaliação do uso de psicotrópicos, construído pelos autores com base em outros estudos ${ }^{(7,8)}$, envolveu as seguintes variáveis: conhecimento sobre a medicação psicotrópica utilizada; uso atual ou prévio de medicação psicotrópica; frequência diária de consumo de psicotrópicos; influência das atividades laborais (docência) no uso de psicotrópicos; uso do medicamento com indicação médica; impacto do medicamento na melhora da qualidade de vida; sentimento de dependência do medicamento; e realização de trabalho de risco. Essas recomendações basearam-se no Centro Brasileiro sobre Drogas Psicotrópicas (CEBRID) ${ }^{(7,8)}$.

Para organização e análise dos dados, criou-se um banco de dados no programa Excel (Office 2018) e utilizou-se o programa Statistical Package for Social Science (SPSS), versão 17,0. As variáveis qualitativas foram apresentadas 
em valores absolutos ( $n$ ) e percentuais (\%) e as variáveis quantitativas, em medidas descritivas: valores mínimos e máximos, média e desvio padrão. Para análise da consistência interna dos instrumentos, utilizou-se o coeficiente alfa de Cronbach. Para análise de associação entre estresse e consumo de psicotrópicos, utilizou-se o teste de qui-quadrado. Valores de $p<0,05$ foram considerados estatisticamente significativos.

Este projeto faz parte de um estudo maior intitulado Fenômenos de saúde e personalidade resiliente em docentes universitários da área da saúde, o que obteve a aprovação do comitê de ética em pesquisa da Faculdade de Ciências e Educação Sena Aires sob o Parecer n. ${ }^{\circ} 2.411 .169$. Além disso, atende às Diretrizes e Normas Regulamentadoras de Pesquisas Envolvendo Seres Humanos (Resolução CNS 466/12). O Termo de Consentimento Livre e Esclarecido foi entregue somente aos docentes que aceitaram participar da pesquisa, junto com os instrumentos, sendo assinado em duas vias (uma para o entrevistado e outra para o pesquisador).

\section{RESULTADOS}

Compuseram a amostra 48 docentes, e os dados de caracterização sociodemográfica entre docentes universitários da área de saúde estão apresentados na Tabela I.

Na Figura 1, apresenta-se a classificação de estresse ocupacional dos docentes universitários da área de saúde.

Tabela I - Caracterização sociodemográfica de docentes universitários da área de saúde. Goiás, 2018.

\begin{tabular}{lcc}
\hline Variáveis sociodemográficas* & n(\%) ou Média (Dp ${ }^{* *}$ ) \\
\hline Sexo (Feminino) & & $25(52,2 \%)$ \\
Idade (Anos) & & $42,5(6,9)^{* *}$ \\
Religião (Católica) & & $21(45,7 \%)$ \\
Renda mensal (4 a 6 salários mínimos) & & $14(31,8 \%)$ \\
Estado civil (Solteiro) & & $23(50,0 \%)$ \\
Escolaridade & Especialização & $17(37,8 \%)$ \\
& Mestrado & $16(35,6 \%)$ \\
Tempo de docência & Acima de 10 anos & $17(37,0 \%)$ \\
& 1 a 5 anos & $13(28,3 \%)$ \\
Tempo de atuação na instituição & Menos de 1 ano & $20(43,5 \%)$ \\
Regime de trabalho (Celetista) & 1 a 5 anos & $14(30,4 \%)$ \\
Jornada semanal de trabalho (Horas) & & $39(84,8 \%)$ \\
Outro vínculo de trabalho (Sim) & & $30(17,0)^{* *}$ \\
Você recebeu treinamento para atuar na IES? (Não) & $31(67,4 \%)$ \\
Principal atividade quando não está trabalhando na IES? (Trabalho em outro local) & $24(50,0 \%)^{\star * *}$ \\
Realização de trabalho de risco (Sim) & & $25(41,0 \%)$ \\
Satisfação no trabalho (Satisfeito) & & $24(52,2 \%)$ \\
\hline
\end{tabular}

*Somente as categorias predominantes para cada variável são apresentadas; ** Desvio-padrão; ** 3 pessoas não responderam à questão. IES: Instituição de ensino superior

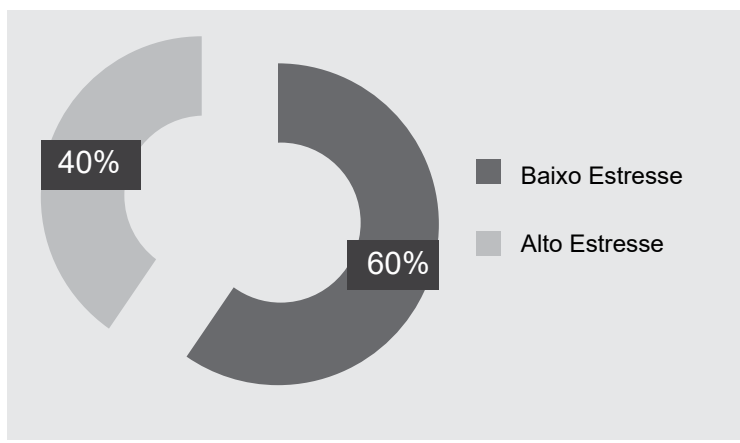

Figura 1 - Classificação de estresse ocupacional dos docentes universitários da área de saúde. Goiás, 2018. 
Na figura, observa-se predomínio de baixo nível de estresse ocupacional entre os docentes universitários, ou seja, $60,0 \%$ dos docentes avaliados apresentam níveis de estresse inferior à média geral para essa população de estudo.

Na Tabela II, apresentam-se os estressores de maior média entre os docentes segundo a EET.

Tabela II - Estressores de maior média entre os docentes universitários da área de saúde segundo a escala de estresse no trabalho (EET). Goiás, 2018.

\begin{tabular}{llc}
\hline Itens (Estressores) & Média & Desvio padrão \\
\hline A forma como as tarefas são distribuídas em minha área tem me deixado nervoso. & 2,7391 & 1,25 \\
Sinto-me incomodado com a falta de informações sobre minhas tarefas no trabalho. & 2,4783 & 1,31 \\
Sinto-me irritado com a deficiência na divulgação de informações sobre decisões & 2,4000 & 1,15 \\
organizacionais. & 2,3721 & 1,17 \\
O tempo insuficiente para realizar meu volume de trabalho deixa-me nervoso. & 2,2826 & 1,11 \\
O tipo de controle existente em meu trabalho me irrita. & \\
\hline
\end{tabular}

Observa-se, na Tabela II, que os itens de maior média e, portanto, que representam maior estresse aos docentes universitários da área de saúde estão relacionados: à distribuição de tarefas; à falta de informações sobre as tarefas recebidas; à deficiência na divulgação de informações relacionadas às decisões da empresa; à percepção de tempo insuficiente para atender às demandas recebidas e à forma de controle dos superiores.

A Tabela III apresenta os resultados da associação entre o nível de estresse ocupacional, a realização de trabalho de risco e o perfil de consumo de psicotrópicos em docentes universitários.

Tabela III - Associação entre o nível de estresse ocupacional, realização de trabalho de risco e o perfil de consumo de psicotrópicos em docentes universitários da área de saúde. Goiás, 2018 (n=48).

\begin{tabular}{|c|c|c|c|c|}
\hline \multirow[b]{2}{*}{ Consumo de psicotrópicos } & \multicolumn{4}{|c|}{ Estresse } \\
\hline & & $\begin{array}{l}\text { Baixo } \\
\text { n (\%) }\end{array}$ & $\begin{array}{l}\text { Alto } \\
\text { n (\%) }\end{array}$ & Valor de $p$ \\
\hline \multirow{2}{*}{ Você sabe o que é uma medicação psicotrópica? } & Sim & $26(57,8 \%)$ & $17(37,8 \%)$ & \multirow{2}{*}{0,987} \\
\hline & Não & $1(2,2 \%)$ & $1(2,2 \%)$ & \\
\hline \multirow{2}{*}{ Uso atual ou prévio de medicação psicotrópica } & Sim & $8(17,8 \%)$ & $11(24,4 \%)$ & \multirow{2}{*}{$0,037^{*}$} \\
\hline & Não & $19(42,2 \%)$ & $7(15,6 \%)$ & \\
\hline \multirow{3}{*}{ Frequência diária de consumo de psicotrópicos** } & 1 vez ao dia & $5(15,6 \%)$ & $8(53,3 \%)$ & \multirow{3}{*}{0,857} \\
\hline & 2 vezes ao dia & $0(0,0 \%)$ & $0(0,0 \%)$ & \\
\hline & 3 vezes ao dia & $1(6,7 \%)$ & $1(6,7 \%)$ & \\
\hline \multirow{2}{*}{$\begin{array}{l}\text { Influência das atividades laborais (docência) no uso } \\
\text { de psicotrópicos }\end{array}$} & Sim & $3(15,8 \%)$ & $4(21,1 \%)$ & \multirow{2}{*}{0,960} \\
\hline & Não & $5(26,3 \%)$ & $7(36,8 \%)$ & \\
\hline \multirow{2}{*}{ Uso do medicamento com indicação médica** } & Sim & $7(36,8 \%)$ & $10(52,6 \%)$ & \multirow{2}{*}{0,810} \\
\hline & Não & $1(5,3 \%)$ & $1(5,3 \%)$ & \\
\hline \multirow{3}{*}{$\begin{array}{l}\text { Impacto do medicamento na melhora da qualidade de } \\
\text { vida }^{* *}\end{array}$} & Sim & $5(26,3 \%)$ & $8(42,1 \%)$ & \multirow{3}{*}{$0,041^{*}$} \\
\hline & Não & $3(15,8 \%)$ & $0(0,0 \%)$ & \\
\hline & Talvez & $0(0,0 \%)$ & $3(15,8 \%)$ & \\
\hline \multirow{3}{*}{ Sentimento de dependência do medicamento** } & Sim & $1(5,3 \%)$ & $1(5,3 \%)$ & \multirow{3}{*}{0,734} \\
\hline & Não & $6(31,6 \%)$ & $7(36,8 \%)$ & \\
\hline & Talvez & $1(5,3 \%)$ & $3(15,8 \%)$ & \\
\hline \multirow{2}{*}{ Realização de trabalho de risco } & Sim & $11(23,9 \%)$ & $13(28,3 \%)$ & \multirow[b]{2}{*}{$0,036^{*}$} \\
\hline & Não & $17(37,0 \%)$ & $5(10,9 \%)$ & \\
\hline
\end{tabular}

* Diferença estatisticamente significativa (Teste de qui-quadrado). ** Quantitativo correspondente apenas aos que utilizam psicotrópico $(n=19)$

Observa-se que há associação significativa entre o uso atual ou prévio de medicação psicotrópica e o nível de estresse no trabalho, de forma que aqueles que não utilizam medicação psicotrópica (42,2\%) possuem um nível de estresse menor $(p=0,037)$. Aqueles que percebem que o medicamento melhora a qualidade de vida apresentam um 
nível de estresse no trabalho superior aos demais $(42,1 \%)(p=0,041)$. Ademais, aqueles que realizam atividades de risco no ambiente de trabalho apresentam um nível de estresse maior $(28,3 \%)$ quando comparados àqueles que não realizam esse tipo de atividade $(10,9 \%)(p=0,036)$.

\section{DISCUSSÃO}

É interessante ressaltar que a maioria dos entrevistados no presente estudo era do sexo feminino, solteiras e de religião católica, o que corrobora um estudo realizado na região metropolitana de Goiânia, Goiás, no qual houve predominância de profissionais do sexo feminino $(54,4 \%)$, solteiras $(35,1 \%)$ e católicas $(50,9 \%)$. Em estudos análogos também foram predominantes o sexo feminino na docência em cursos da área da saúde, o que é um fator preocupante, pois as condições e os fatores estressores no sexo feminino são mais inerentes devido à carga laboral cumulativa que une as primícias familiares associadas às demandas profissionais, o que justifica, muitas vezes, a possibilidade de adoecimento neuropsicossocial, prejudicando o rendimento laboral, o desnovelar das práticas diárias e o enfrentamento dos problemas organizacionais ${ }^{(10,11)}$.

No presente estudo, a medicação assume um processo terapêutico que se torna irreal, sendo percebido pelo docente como um benefício, uma ajuda no controle dos seus sentimentos atribuídos à atividade laboral. Mas, no que se diz respeito ao estresse, observa-se que as medicações não contribuem para a redução do estresse de forma significativa. Outros estudam apontam, sobre o efeito dessa medicação na vida desse indivíduo, que trazem malefícios em longo e curto prazo, mais a literatura não discute esses efeitos sobre a ótica do profissional docente ${ }^{(8,12-14)}$.

Percebe-se que na IES superior pesquisada os docentes estão atuando há menos de 1 ano (43,5\%), contrapostos a outros funcionários que já operam há mais de 10 anos (37\%), com jornada média de 30 horas semanais, em regime de trabalho celetista $(84,8 \%)$. Esse paralelo de funcionários novos opostos aos funcionários antigos demonstra que a IES pesquisada está passando por mudanças contínuas no último ano e que essas mudanças podem ocasionar nos colaboradores de maior tempo estresse e medo de desligamento devido a não adaptação às novas primícias educacionais, acarretando prejuízos nas práticas laborais diárias ${ }^{(2)}$.

Dos entrevistados na atual pesquisa, $67,4 \%$ possuem outro vínculo de trabalho, cujo patamar se justifica pelas tentativas de manter um padrão de vida confortável financeiramente, de modo que a maior parte dos profissionais da educação e saúde acabam tendo mais de um vínculo empregatício, o que, consequentemente, aumenta sua carga horária de trabalho, sendo este um dos principais agentes que contribui para o estresse, pois quanto mais tempo o profissional é submetido ao ambiente incitador do estresse, maior é sua chance de adoecer(2,5).

Em geral, o trabalho e o conhecimento do docente são levados em consideração em avaliações curriculares, as quais esses profissionais são submetidos pelas IES. Porém precisam ter uma menor carga horária e limitar as suas atividades, tendo a garantia do auxílio financeiro e da valorização do seu trabalho, inclusive quando a busca de titulação estiver envolvida ${ }^{(11)}$.

Encontrou-se que $50 \%$ dos docentes disseram não ter recebido treinamento para atuar na IES. O não recebimento de treinamento causa distúrbios de ansiedade ocasionados por algo não conhecido até então, fruto do medo do novo e do que será enfrentado, sem uma direção e capacitação prévia no ambiente corporativo, evidenciado pela profissão ser apontada por diversas pesquisas na literatura global como uma das profissões mais estressantes na atualidade e se tornando um problema de saúde pública relacionado aos problemas de caráter ocupacional, advindos de profissionais adoecidos que formaram outros ${ }^{(2)}$.

Entre os docentes investigados na presente pesquisa, $73,9 \%$ estão satisfeitos em relação ao trabalho desenvolvido, apesar dos dados evidenciarem um quantitativo de docentes com alto nível de estresse, que pode estar relacionado ao uso de medicação psicotrópica, pois $42,1 \%$ dos que fazem o uso desse tipo de medicamento disseram que a medicação melhorou sua qualidade de vida. O baixo nível de estresse ajuda a melhorar a satisfação pessoal e profissional, com o aumento do desempenho em relação ao trabalho ${ }^{(12)}$.

O entendimento do comportamento humano no ambiente corporativo tornou-se relevante, pois se o trabalhador não estiver satisfeito, isto pode afetar diretamente na qualidade do serviço prestado e na sua própria saúde. Isto ocorre devido ao grau de satisfação e motivação do profissional, que é considerado um motivo que pode afetar a harmonia e a estabilidade psicológica no ambiente de trabalho ${ }^{(13)}$.

Observou-se que, dentre os docentes universitários, 39,6\% deles apresenta um nível de estresse elevado em comparação à média do total dos docentes pesquisados. Esse quantitativo é bastante elevado, representando quase metade do total. Os profissionais que são subordinados às altas demandas psicológicas, somado ao baixo autocontrole diante do trabalho, possuem um risco maior de desenvolver patologias de caráter físico e mental devido aos fatores estressantes, que se dão graças ao grande desgaste no trabalho ou, até mesmo, grande autoexigência. 
No local em que os níveis de estresse são elevados recomenda-se uma política de promoção de saúde que vise o bem-estar psicológico e biológico dos trabalhadores ${ }^{(14)}$.

Os fatores dificultosos elencados no estudo foram em relação: a forma como as tarefas são distribuídas em minha área tem me deixado nervoso. Nos últimos anos, no Brasil, houve um considerável crescimento do ensino superior privado, entretanto à custa de muitas modificações na rotina do trabalho do docente, o que justifica esses resultados, além da falta de preparo dos novos contratados oriundos de programas de pós-graduação stricto e lato senso sem o devido preparo para a docência(15).

Outros fatores dificultadores relevantes em relação aos docentes foram: sentirem-se incomodados com a falta de informações sobre as tarefas no trabalho; sentirem-se irritados com a deficiência na divulgação de informações sobre decisões organizacionais; e em relação ao tempo, considerado insuficiente para realizar o volume de trabalho, ocasionando nervosismo. É importante salientar que a criação de espaços para que o trabalhador possa compartilhar experiências e vivências do dia a dia nas organizações é extremamente benéfica, pois ocasiona um melhor entendimento do que sentem perante as dificuldades enfrentadas ${ }^{(16)}$.

Do ponto de vista gerencial, percebe-se a importância de se construir na organização um clima de suporte social, um ambiente relacional positivo no trabalho, tanto entre colegas como entre subordinados e gerentes. Nesse sentido, é necessário que as organizações de ensino superior, em especial as da área da saúde, desenvolvam práticas de gestão que adotem a efetiva participação dos trabalhadores nos processos de trabalho, de forma a possibilitar o bem-estar e a promoção da saúde psíquica ${ }^{(17)}$.

O tipo de controle existente no trabalho também irritou os investigados e também representou um fator determinante, de modo que a insatisfação se diferencia em cada nível ocupacional, estando relacionada com o sentido de autonomia e controle que o profissional tem ao desempenhar suas tarefas, sendo um dos principais fatores de insatisfação a insuficiência de possibilidade de participação e as dificuldades para controlar suas próprias tarefas ${ }^{(13-18)}$.

Em relação à associação entre o nível de estresse ocupacional, a realização de trabalho de risco e o perfil de consumo de psicotrópicos, observou-se, na atual pesquisa, que existe uma associação significativa entre o uso (atual ou prévio) de medicação psicotrópica e o nível de estresse no trabalho, de forma que aqueles não utilizam medicação psicotrópica $(42,2 \%)$ possuem um nível de estresse menor $(p=0,037)$.

O nível de estresse pode tornar o profissional incapacitado, pois a pressão e o estresse vivenciado em seu ambiente de trabalho não impedem que o profissional queira dar o seu melhor para garantir seu emprego, mesmo frente à sobrecarga de trabalho, conflitos, desvalorização profissional e dupla jornada de trabalho, mas todos esses fatores contribuem para o profissional fazer uso de substâncias psicoativas ${ }^{(19,20)}$.

Aqueles que perceberam que o medicamento melhora a qualidade de vida apresentaram um nível de estresse no trabalho superior aos demais $(42,1 \%)(p=0,041)$. Segundo o Ministério da Saúde, pelo menos 23 milhões de pessoas no Brasil usam ou usarão, pelo menos uma vez, medicamentos de caráter psicotrópico, cedidos pelos serviços de saúde mental(21).

Observou-se, ainda, que os docentes que consideraram que realizam atividades de risco no ambiente de trabalho apresentam um nível de estresse maior $(28,3 \%)$ quando comparados àqueles que não têm essa percepção quanto a esse tipo de atividade (10,9\%). Os riscos causados pelo estresse podem ser entendidos de várias formas, não sendo levado em consideração apenas a integridade física do indivíduo, mas também seus fatores psicológicos e a sua saúde física. O ser humano é levado a sofrer danos quando se submete a uma grande carga de agentes estressores, e isto pode ocasionar consequências terríveis se não tratados com o devido cuidado ${ }^{(20-23)}$. Além do tratamento medicamentoso, recomenda-se um período de lazer, entendido e percebido como sendo uma ação primordial na promoção da saúde e na redução do estresse ocupacional do docente.

Logo, o uso exacerbado de medicamentos e a polimedicação podem ser fatores preditores para a diminuição da qualidade de vida e da longevidade, devido ao desconhecimento dos efeitos adversos. Nesse contexto, esses fatores se exibem como um problema de saúde pública, sendo necessária a implantação de políticas públicas efetivas e inerentes para a diminuição da medicalização e a valorização da utilização de métodos alternativos, proporcionando o enfrentamento das práxis vivenciadas e a melhora significativa do indivíduo em sofrimento moral ${ }^{(24-26)}$.

O estresse excessivo pode ser, também, um risco para a sobrevivência de empresas, pois os custos operacionais se elevam através da queda de produtividade, dos acidentes causados no ambiente de trabalho, do desperdício de materiais, do aumento da ausência de colaboradores e do significativo gasto com assistência médica ${ }^{(20)}$.

As limitações do estudo se deram devido a publicações insuficientes referentes ao adoecimento ocupacional de docentes, inviabilizando a discussão de forma ampla e fundamentada em dados epidemiológicos recentes acerca dos fatores estressores relacionados à temática transcorrida. 
Urge, portanto, a necessidade de medidas que possam sanar o uso de medicações psicotrópicas e reduzir o estresse ocupacional dos profissionais da saúde que atuam na educação superior, sugerindo-se novos trabalhos nessa área.

\section{CONCLUSÃO}

O uso de psicotrópicos pelos docentes universitários investigados está significativamente associado ao estresse ocupacional, de maneira que docentes com maior nível de estresse fazem uso de medicação psicotrópica no presente ou já fizeram.

\section{CONTRIBUIÇÕES}

Todos os autores contribuíram com a elaboração e delineamento do estudo, aquisição, análise e interpretação dos dados, e redação e/ou revisão do manuscrito.

\section{REFERÊNCIAS}

1. Brasil. Decreto lei $n^{\circ} 10.861$, de 04 de 14 de abril de 2004. Institui o Sistema Nacional de Avaliação da Educação Superior - SINAES e dá outras providências [Internet]. Brasília, 14 de abril de 2004 [aceso em 2018 Set 25]. Disponível em: http://www.planalto.gov.br/ccivil_03/_ato2004-2006/2004/lei//10.861.htm.

2. Araújo BLS, Gomes DV, Pires VS, Moraes IM Filho, Costa ALS. Estresse ocupacional em docentes de uma Instituição de Ensino Superior da região metropolitana de Goiânia. REVISA. 2015;4(2):22-30.

3. Ministério da Educação (BR). Instrumento de Avaliação de Cursos de Graduação presencial e a distância [Internet]. Brasília: Ministério da Educação; 2016 [acesso em 2018 Set 25]. Disponível em: http://cpa.ufsc.br/ files/2017/02/Instrumento-Curso-2016.pdf

4. Sandor S, Yvette T, Arpad S. The legacy of Hans Selye and the origins of stress research: a retrospective 75 years after his landmark brief "letter" to the editor\# of nature. Stress [Internet]. 2012 [acesso em 2018 Dez 22];15(5):472-8. Disponível em: http://selyeinstitute.org/wp-content/uploads/2013/06/ TheLegacuyofHansSelyearticle.pdf

5. Lima MFEM, Lima DOF. Condições de trabalho e saúde do/a professor/a universitário/a. Ciênc Cogn. 2009;14(3):62-82.

6. Moran R. Retention of new graduate nurses. J Nurses Staff Dev. 2012;28(6):270-3.

7. Borges TL, Miasso Al, Vedana KGG, Telles PCP Filho, Hegadoren KM. Prevalência do uso de psicotrópicos e fatores associados na atenção primária à saúde. Acta Paul Enferm [Internet]. 2015 [acesso em 2018 Dez 08];28(4):344-9. Disponível em: http://www.scielo.br/scielo.php?script=sci_arttext\&pid=S0103$21002015000400009 \& \operatorname{lng}=e n$

8. Bezerra IC, Morais JB, Paula ML, Silva TMR, Jorge MSB. Uso de psicofármacos na atenção psicossocial: uma análise à luz da gestão do cuidado. Saúde Debate [internet]. 2016 [acesso em 2018 Dez 08];40(110):148-61. Disponível em: https://doi.org/10.1590/0103-1104201611011

9. Paschoal T, Tamayo A. Validação da escala de estresse no trabalho. Estud Psicol (Campinas). 2004;9(1):4552.

10. Morero JAP, Bragagnollo GR, Santos MTS. Estratégias de enfrentamento: uma revisão sistemática sobre instrumentos de avaliação no contexto brasileiro. Rev Cuid [Internet]. 2018 [acesso em 2019 Jan 15];9(2):2257-68. Disponível em: http://dx.doi.org/10.15649/cuidarte.v9i2.503

11. Sims J. Nursing Faculty Shortage in 2009. Dimens Crit Care Nurs. 2009;28(5):221-3.

12. Rocha BS, Werlang MC. Psicofármacos na estratégia saúde da família: perfil de utilização, acesso e estratégias para a promoção do uso racional. Ciênc Saúde Colet [Internet]. 2013 [acesso em 2017 Ago 28];18(11):3291-300. Disponível em: http://www.scielo.br/pdf/csc/v18n11/19.pdf

13. Ferreira ACM, Brasil VV, Zatta LT, Moraes KL, Soares LR, Santos LF. Satisfação no trabalho de docentes de ensino superior na área da saúde. Saúde Ciên Ação. 2016;2(01):93-30. 
14. Schmidt DRC. Modelo Demanda-Controle e estresse ocupacional entre profissionais de enfermagem: revisão integrativa. Rev Bras Enferm [Internet]. 2013 [acesso em 2018 Dez 08];66(5):779-88. doi: http://dx.doi. org/10.1590/S0034-71672013000500020

15. Gontijo MR, Garcia FC. Mercantilização do ensino: a percepção dos docentes do ensino superior privado no Brasil. Rev Fac Adm Economia [Internet]. 2017 [acesso em 2018 Dez 08];8(2):60-86. Disponível em: https:// www.metodista.br/revistas/revistas-ims/index.php/ReFAE/article/view/7486

16. Bellenzani R, Paro DM, Oliveira MC. Trabalho em saúde mental e estresse na equipe: questões para a política nacional de humanização/SUS. Rev Psicol Saúde [Internet]. 2016 [acesso em 2018 Dez 08];8(1):3243. doi: http://dx.doi.org/10.20435/2177093X2016105

17. Stefano SR, Bonanato FM, Raifur L. Estresse em funcionários de uma instituição de ensino superior: Diferenças entre gênero. Rev Econ Gest. 2013;13(31):73-92.

18. Sá SCA, Silva RM, Kimura CA, Pinheiro GQ, Guido LA, Moraes IM Filho. Estresse em docentes universitários da área de saúde de uma faculdade privada do entorno do Distrito Federal. REVISA. 2018;7(3):200-7.

19. Fernandes MA, Silva JS, Vilarinho JOV, Seabra LO, Feitosa CDA. Use of psychoactive substance by health professionals: integrative Review. SMAD Rev Eletrônica Saúde Mental Alcool Drog [Internet]. 2017 [acesso em 2019 Jan 23];13(4):221-31. Disponível em: https://www.redalyc.org/articulo.oa?id=80356417007

20. Koch MO, Biazi RJ, Benedetto C. Estresse em docentes: um estudo comparativo entre uma instituição de ensino superior pública e uma instituição de ensino superior privada na cidade de Toledo-PR. Revista Uningá. 2015;21(1):17-23.

21. Prado MAMB, Francisco PMSB, Barros MBA. Uso de medicamentos psicotrópicos em adultos e idosos residentes em Campinas, São Paulo: um estudo transversal de base populacional. Epidemiol Serv Saúde [Internet]. 2017 [acesso em 2018 Dez 08];26(4):747-58. Disponível em: http://www.scielo.br/scielo. php?pid=S2237-96222017000400747\&script=sci_abstract\&tlng=pt

22. Sottimano I, Guidetti G, Converso D, Viotti S. We cannot be "forever young," but our children are: a multilevel intervention to sustain nursery schoolteachers' resources and well-being during their long work life cycle. PLos ONE [Internet]. 2018 [acesso em 2018 Dez 18];13(11):1-17. doi: https://doi.org/10.1371/journal. pone.0206627

23. Rosa BN, Milhomem AHCS, Moraes IM Filho, Santos OP, Frasca LLM, Fidelis A. Florescimento organizacional e rotatividade. Rev Inicaç Cient Extensão. 2019;2(2):8-12.

24. Nascimento RCRM, Álvares J, Guerra AA Jr, Gomes IC, Silveira MR, Costa EA, et al. Polifarmácia: uma realidade na atenção primária do Sistema Único de Saúde. Rev Saúde Pública [Internet]. 2017 [acesso em 2019 Set 13];51(Suppl 2):19s. doi: http://dx.doi.org/10.11606/s1518-8787.2017051007136

25. Moraes IM Filho, Almeida RJ. Estresse ocupacional no trabalho em enfermagem no Brasil: uma revisão integrativa. Rev Bras Promoç Saúde [Internet]. 2016 [acesso em 2018 Dez 18];29(3):447-54. Disponível em: https://periodicos.unifor.br/RBPS/article/view/4645

26. Cassoni TCJ, Corona LP, Romano-Lieber NS, Secoli SR, Duarte YAO, Lebrão ML. Uso de medicamentos potencialmente inapropriados por idosos do Município de São Paulo, Brasil: Estudo SABE. Cad Saúde Pública [Internet]. 2014 [acesso em 2019 Set 13];30(8):1708-20. doi: http://dx.doi.org/10.1590/0102$311 \times 00055613$.

\author{
Endereço para correspondência: \\ lel Marciano de Moraes Filho \\ Universidade Paulista - UNIP \\ Campus Brasília - Depertamento de Enfermagem \\ Sgas, Quadra 913, s/n - Conjunto B - Asa Sul \\ CEP: 70390-130 - Brasília - DF - Brasil \\ E-mail: ielfilho@yahoo.com.br
}

Como citar: Moraes IM Filho, Dias CCS, Pinto LL, Santos OP, Félis KC, Proença MFR, et al. Associação de estresse ocupacional e uso de psicotrópicos por docentes da área da saúde. Rev Bras Promoç Saúde. 2019;32:9007. 